\title{
RINGS WITH FLAT SOCLES
}

\author{
YUFEI XIAO \\ (Communicated by Ken Goodearl)
}

\begin{abstract}
We show that the class of rings with flat socles is closed under the formation of polynomial extensions, direct products, and excellent extensions. We also point out that if a ring $R$ can be embedded into a semisimple Artinian ring, then all finitely generated flat $R$-modules are projective.
\end{abstract}

Rings with projective socles are studied by W. K. Nicholson and J. F. Watters [5]. They show that the class of rings with projective right socles is closed under the formation of polynomial extensions, direct products, and matrix rings. They also prove that this property is Morita invariant. Motivated by their work, we study the rings with flat socles. First we give an example of a ring with a non-projective flat right socle. Then we show that the class of rings with flat right socles has the same properties listed above as the class of rings with projective right socles. We also show that the excellent extension of a ring with flat right socle is again a ring with flat right socle. Finally, we show that under some weak chain conditions, a ring with flat right socle must be a ring with projective right socle. Our discussions also show that if a ring $R$ can be embedded into a semisimple Artinian ring, then all finitely generated flat $R$-modules are projective.

All rings throughout this paper are associative and have identities. A ring $R$ satisfies $P D C C^{\perp}$ (the descending chain condition on the principle right annihilators) if there does not exist a properly descending infinite chain: $r\left(x_{1}\right)>$ $r\left(x_{2}\right)>\cdots>r\left(x_{n}\right)>\cdots$, for any sequence $\left\{x_{n}\right\}_{1}^{\infty} \subset R$. Similarly we may define $P A C C^{\perp},{ }^{\perp} P D C C$, and ${ }^{\perp} P A C C$. A ring $R$ satisfies left $P A C C$ (the ascending chain condition on the principal left ideals) if there does not exist a properly ascending infinite chain: $R x_{1}<R x_{2}<\cdots<R x_{n}<\cdots$, for any sequence $\left\{x_{n}\right\}_{1}^{\infty} \subset R$. Similarly we may define right $P A C C$ and left (right) $P D C C$. Clearly the rings satisfying left (right) $P D C C$ are just right (left) perfect rings. When $R_{R}$ is $p$-injective (i.e., any $R$-homomorphism from a principal right ideal of $R$ to $R_{R}$ can be extended to an $R$-homomorphism from $R_{R}$ to $R_{R}$ ), it is easy to show that $R$ satisfies $P A C C^{\perp}$ (resp. $P D C C^{\perp}$ ) if and only if $R$ satisfies left $P D C C$ (resp. right $P A C C$ ). Therefore, speaking roughly, we say that $P A C C^{\perp}$ is the dual of left $P D C C$, etc.

Let $I$ be a right ideal of a ring $R$. Then $(R / I)_{R}$ is flat if and only if $x \in I x$

Received by the editors September 21, 1993 and, in revised form, December 27, 1993.

1991 Mathematics Subject Classification. Primary 16D40; Secondary 16E50. 
for every $x \in I$ (see $[1,19.18]$ ). We will frequently use this property. A ring $S$ is an excellent extension of a ring $R$ if

(1) $S$ is a free normalizing extension of $R$ with a basis that includes 1 ; that is, there are finitely many elements $s_{1}, \ldots, s_{n} \in S$ such that $s_{1}=1$, $S=R s_{1}+\cdots+R s_{n}, R s_{i}=s_{i} R$ for all $i=1, \ldots, n$ and $S$ is free with basis $\left\{s_{1}, \ldots, s_{n}\right\}$ as both a right and left $R$-module.

(2) $S$ is right $R$-projective; that is, if $M_{S}$ is a submodule of $N_{S}$ and $M_{R}$ is a direct summand of $N_{R}$, then $M_{S}$ is a direct summand of $N_{S}$.

A right $R$-module $M_{R}$ is called a $P S$-module if $\operatorname{Soc}\left(M_{R}\right)$ is projective. A ring $R$ is called a right $P S$-ring if $R_{R}$ is a $P S$-module (see [5]). Here we define a right $R$-module $M_{R}$ to be an $F S$-module if $\operatorname{Soc}\left(M_{R}\right)$ is flat. A ring $R$ is a right $F S$-ring if $R_{R}$ is an $F S$-module. As we know, all semiprime rings, right $p . p$. rings, right nonsingular rings, and $V$-rings are right $P S$-rings and therefore right $F S$-rings [5]. Here we mention that right $S F$-rings (rings whose simple modules are flat) are right $F S$-rings. The following example, which is due to Victor Camillo, shows that a right $F S$-ring may not be a right $P S$-ring.

Example 1. Let $A$ be a simple non-Artinian regular ring and $S_{A}=A / M$, where $M$ is a maximal right ideal of $A$. Denote $D=\operatorname{End}_{A}(S)$, a division ring, and set

$$
R=\left[\begin{array}{cc}
A & 0 \\
{ }_{D} S_{A} & D
\end{array}\right]
$$

Then $\operatorname{Soc}\left(R_{R}\right)$ is flat, but it is not projective.

Proof. Since ${ }_{D} S$ is faithful, from [3] we see that

$$
\operatorname{Soc}\left(R_{R}\right)=\left[\begin{array}{ll}
\operatorname{Soc}\left(A_{A}\right) & 0 \\
\operatorname{Soc}\left(S_{A}\right) & 0
\end{array}\right]=\left[\begin{array}{ll}
0 & 0 \\
S & 0
\end{array}\right]=\left[\begin{array}{ll}
0 & 0 \\
1 & 0
\end{array}\right] \cdot\left[\begin{array}{ll}
A & 0 \\
S & D
\end{array}\right]
$$

where $\overline{1}=1_{A}+M \in A / M$. Let $x=\left[\begin{array}{ll}0 & 0 \\ 1 & 0\end{array}\right]$. Then $\operatorname{Soc}\left(R_{R}\right)=x R$. Since $r(x)=\left[\begin{array}{cc}M & 0 \\ S & D\end{array}\right]$ and $M_{A}$ is not a direct summand of $A_{A}, r(x)$ is not a direct summand of $R_{R}$. Therefore $\operatorname{Soc}\left(R_{R}\right)=x R$ is not projective.

Consider the following exact sequence

$$
0 \rightarrow r(x) \rightarrow R \rightarrow x R \rightarrow 0 .
$$

For every $\alpha=\left[\begin{array}{cc}m & 0 \\ s & d\end{array}\right] \in r(x)$, take $y \in A$ such that $m=m y m$ and set $\beta=\left[\begin{array}{cc}m y & 0 \\ 0 & 1\end{array}\right] \in r(x)$. Then

$$
\beta \alpha=\left[\begin{array}{cc}
m y & 0 \\
0 & 1
\end{array}\right] \cdot\left[\begin{array}{cc}
m & 0 \\
s & d
\end{array}\right]=\left[\begin{array}{cc}
m y m & 0 \\
s & d
\end{array}\right]=\alpha .
$$

Therefore $\operatorname{Soc}\left(R_{R}\right)=x R$ is flat.

Comparing to [5, 2.4], we have:

Theorem 2. For a ring $R$, the following are equivalent:

(1) $R$ is a right FS-ring.

(2) $R$ has a faithful right FS-module.

(3) For every maximal right ideal $M$, either $l(M)=0$ or $(R / M)_{R}$ is flat.

(4) For every simple right $R$-module $T_{R}$, either $T_{R}$ is flat or ${ }_{R} T^{*}=$ $\operatorname{Hom}_{R}(T, R)=0$.

The following example shows that the notation of an $F S$-ring is not left-right symmetric. 
Example 3. Let $S$ be a semiprimary ring which is not a left $F S$-ring (for example, a ring with only one proper left ideal $\left.\left[2,7.11^{\prime} .1\right]\right)$. Assume that $J(S)^{n} \neq 0$, $J(S)^{n+1}=0$. Set $D=S / J(S)$ and $R=\left[\begin{array}{cc}D & 0 \\ J^{n} & S\end{array}\right]$. Then $R$ is a right $F S$-ring which is not a left $F S$-ring.

Proof. It is easy to see that

$$
\operatorname{Soc}\left(R_{R}\right)=\left[\begin{array}{cc}
\operatorname{Soc}\left(D_{D}\right) & 0 \\
\operatorname{Soc}\left(\left(J^{n}\right)_{D}\right) & 0
\end{array}\right]=\left[\begin{array}{cc}
D & 0 \\
J^{n} & 0
\end{array}\right]
$$

which is projective from [3,4.5]. Thus $R$ is a right $F S$-ring. Since $S$ is not a left $F S$-ring, from (3) of Theorem $2 R$ is not a left $F S$-ring.

Proposition 4. Let $R=\prod_{i \in I} R_{i}$ be a direct product of rings. Then $R$ is a right $F S$-ring if and only if each $R_{i}$ is a right FS-ring.

Proof. From Theorem 2 and [5, 3.3].

Proposition 5. If $R$ is a right $F S$-ring, then $R[x]$ is also a right $F S$-ring.

Proof. Take a maximal right ideal $M$ of $R[x]$ and let $I$ denote the left ideal of $R$ consisting of all trailing coefficients of polynomials in $M$ with 0 adjoined.

Case 1: If $I=R$, then $l(M)=0$.

Case 2: If $I \neq R$, we first claim that $I$ is a maximal right ideal of $R$ and $I \subset M$. For every $a \in R-I$, we have that $a \notin M$, so that $M+a R[x]=R[x]$, which implies that there exists an $m_{0} \in I$ such that $m_{0}+a r=1$ for some $r \in R$, thus $I+a R=R$. Therefore $I$ is a maximal right ideal of $R$. If there is an $i \in I-M$, then $M+i R[x]=R[x]$, which implies that $I+i R=R$ and so $I=R$, a contradiction. Therefore $I \subset M$.

Now assume that $l(M) \neq 0$. Then, because $I \subset M$, we have $l(I) \neq 0$. Thus $(R / I)_{R}$ is flat from Theorem 2. We need to show that $(R[x] / M)_{R[x]}$ is flat. For each polynomial $f \in R[x]$, let $|f|$ denote the number of non-zero coefficients of $f$. Take

$$
f(x)=b_{0} x^{n}+b_{1} x^{n+1}+\cdots+b_{k} x^{n+k} \in M
$$

with $b_{0} \neq 0$. Since $b_{0} \in I$, there exists an $i_{0} \in I$ such that $b_{0}=i_{0} b_{0}$, i.e. $\left(1-i_{0}\right) b_{0}=0$. Next denote

$$
f_{1}(x)=f(x)-i_{0} f(x)=\left(1-i_{0}\right) f(x) .
$$

Then $f_{1}(x) \in M$ and $\left|f_{1}\right|<|f|$. Repeating this process, we find $i_{0}, i_{1}, \ldots, i_{k}$ $\in I \subset M$ such that

$$
0=\left(1-i_{k}\right)\left(1-i_{k-1}\right) \cdots\left(1-i_{0}\right) f(x) .
$$

Thus $f=\sigma f$, where $\sigma=1-\prod_{j=0}^{k}\left(1-i_{j}\right) \in I \subset M$. Therefore $(R[x] / M)_{R[x]}$ is flat.

Proposition 6. Let $R$ and $S$ be Morita equivalent rings. If $R$ is a right FS-ring, then so is $S$.

Proof. Let $F: \mathbf{M}_{R} \rightarrow \mathbf{M}_{S}$ be an equivalence map and set $F(R)=P$. Then from $[1,22.1] P_{S}$ and ${ }_{R} P$ are progenerators and $F \cong\left({ }_{-} \otimes_{R} P\right)$. This together with $[1,21.7]$ implies that $\operatorname{Soc}\left(P_{S}\right)$ is flat. Therefore $\operatorname{Soc}\left(S_{S}\right)$ is flat.

The above proposition implies that the matrix ring over a right $F S$-ring is also a right $F S$-ring. Moreover, we have 
Proposition 7. Let $S$ be an excellent extension of a ring $R$. Then

(1) $S$ is a right $F S$-ring if and only if $R$ is a right $F S$-ring.

(2) If $R$ is a left $F S$-ring, then so is $S$.

Proof. (1) From [6, 1.3] we have

$$
\operatorname{Soc}\left(R_{R}\right) \otimes_{R} S \cong \operatorname{Soc}\left(R_{R}\right) S=\operatorname{Soc}\left(S_{S}\right) .
$$

Therefore from [8] we see that $\operatorname{Soc}\left(R_{R}\right)$ is flat if and only if $\operatorname{Soc}\left(S_{S}\right)$ is flat.

(2) From [10, Remark 1] we have

$$
\operatorname{Soc}\left({ }_{S} S\right) \subseteq S \cdot \operatorname{Soc}\left({ }_{R} R\right) \cong S \otimes_{R} \operatorname{Soc}\left({ }_{R} R\right) \text {. }
$$

Since $\operatorname{Soc}\left({ }_{R} R\right)$ is flat, ${ }_{R}\left(S \cdot \operatorname{Soc}\left({ }_{R} R\right)\right)$ is flat. Thus ${ }_{R} \operatorname{Soc}\left({ }_{S} S\right)$ is flat because ${ }_{R}\left(S \cdot \operatorname{Soc}\left({ }_{R} R\right)\right)$ is semisimple. So $\left(\operatorname{Soc}\left({ }_{S} S\right)\right)_{R}^{*}$, the character module of ${ }_{R} \operatorname{Soc}\left({ }_{S} S\right)$, is injective, which implies that $\left(\operatorname{Soc}\left({ }_{S} S\right)\right)_{S}^{*}$ is injective. Therefore ${ }_{s} \operatorname{Soc}\left({ }_{s} S\right)$ is flat.

Now we give some conditions under which a right $F S$-ring must be a right $P S$-ring.

Proposition 8. If a right $F S$-ring $R$ satisfies any one of the following conditions, then $R$ is a right $P S$-ring.

(1) $R$ is a commutative ring.

(2) $R$ satisfies $P A C C^{\perp}$.

(3) $r$ satisfies ${ }^{\perp} P D C C$.

(4) $R$ satisfies right $P A C C$, in particular, if $R$ is a right Noetherian or right Artinian ring.

Proof. (1) Let $x R$ be a minimal right ideal of $R$ which is flat but not projective. Then $r(x)$ is essential in $R_{R}$, which implies that $r(x) \cap x R \neq 0$. Thus there exists an $r \in R$ such that $0 \neq x r \in r(x)$. But then since $x R$ is flat, there is an $m \in r(x)$ such that $x r=m(x r)$, which implies that $x r=(m x) r=0 r=0$, a contradiction. Therefore $R$ must be a right $P S$-ring.

(2)-(4) If $R$ satisfies any one of these conditions, then from [12] (or [11, 1.3]) all cyclic flat right $R$-modules are projective. Therefore $R$ is a right $P S$ ring.

The following lemma is basically from [4]:

Lemma 9. Let a ring $R$ be a subring of a ring $S$. For a finitely generated flat right $R$-module $M_{R}$, if $M \otimes_{R} S$ is projective as a right $S$-module, then $M_{R}$ is projective.

Proof. Take an exact sequence

$$
0 \rightarrow K_{R} \rightarrow F_{R} \rightarrow M_{R} \rightarrow 0
$$

where $F_{R}$ is a finitely generated free $R$-module. Since $M_{R}$ is flat, the above sequence is pure, which implies an exact sequence

$$
0 \rightarrow K \otimes_{R} S \rightarrow F \otimes_{R} S \rightarrow M \otimes_{R} S \rightarrow 0 .
$$

Since $\left(M \otimes_{R} S\right)_{S}$ is projective, $K \otimes_{R} S$ is a direct summand of $F \otimes_{R} S$ and so is finitely generated. Let $\left\{k_{i} \otimes 1\right\}_{1}^{n}$ be a finite set of $S$-generators of $K \otimes_{R} S$. From [7, 3.57] there exists an $R$-homomorphism $\theta: F \rightarrow K$ such that $\theta\left(k_{i}\right)=k_{i}$ for every $i$. Now for every $k \otimes s=\sum_{i=1}^{n} k_{i} \otimes s_{i} \in K \otimes_{R} S$

$$
\theta(k) \otimes s=\sum \theta\left(k_{i}\right) \otimes s_{i}=\sum k_{i} \otimes s_{i}=k \otimes s .
$$


Set $\lambda=\theta(k)-k$. Then $\lambda \otimes s=0$ for all $s \in S$. Especially $\lambda \otimes 1=0$. Noticing that $\lambda \in F, \lambda$ must have the form $\lambda=\sum e_{i} r_{i}$, where $\left\{e_{i}\right\}_{1}^{t}$ is a free basis of $F$. Thus we have $\sum e_{i} \otimes r_{i}=0$, and so each $r_{i}=0$, which implies that $\lambda=0$. This means that $\theta(k)=k$ for each $k \in K$. Therefore $M_{R}$ is projective.

Corollary 10. If a right (left) FS-ring $R$ can be embedded into a semisimple Artinian ring, then $R$ is also a right (left) PS-ring.

Corollary 11. If a ring $R$ can be embedded into a semisimple Artinian ring, then all finitely generated flat $R$-modules are projective.

Corollary 12 (Stewart [9]). Let a ring $S$ be an excellent extension of a ring $R$. Then $S$ is a right PS-ring if and only if $R$ is a right $P S$-ring.

Proof. First assume that $S$ is a right $P S$-ring. Take a minimal right ideal $T$ of $R$. Observe that

$$
\left(T \otimes_{R} S\right)_{S} \cong T \cdot S \leq \operatorname{Soc}\left(S_{S}\right),
$$

$\left(T \otimes_{R} S\right)_{S}$ is projective as well as flat. Thus from [8] $T_{R}$ is flat, which implies that $T_{R}$ is projective from Lemma 9 .

Conversely assume that $R$ is a right $P S$-ring. From [6] we have

$$
\operatorname{Soc}\left(R_{R}\right) \otimes S \cong \operatorname{Soc}\left(R_{R}\right) \cdot S=\operatorname{Soc}\left(S_{S}\right) \text {. }
$$

Therefore from the well-known Adjoint Associativity Theorem (see [1, 21.7]) $\operatorname{Soc}\left(S_{S}\right)$ is projective.

\section{ACKNOWLEDGMENT}

The author expresses his sincere appreciation to professor Victor Camillo for his helpful advice.

\section{REFERENCES}

1. F. W. Anderson and K. R. Fuller, Rings and categories of modules, Springer-Verlag, New York, 1974.

2. C. Faith, Algebra. I, Rings, Modules, and Categories, Springer-Verlag, Berlin, Heidelberg, and New York, 1981.

3. K. R. Goodearl, Ring theory, Nonsingular Rings and Modules, Marcel Dekker, New York and Basel, 1976.

4. S. Mangauarcarassy and T. Duraivel, $A$ note on flat modules and a theorem of Jondrup, Comm. Algebra 21 (1993), 1421-1426.

5. W. K. Nicholson and J. F. Watters, Rings with projective socle, Proc. Amer. Math. Soc. 102 (1988).

6. M. M. Parmenter and P. N. Stewart, Excellent extensions, Comm. Algebra 16 (1989), 703-713.

7. J. J. Rotman, An introduction to homological algebra, Academic Press, New York, 1979.

8. A. Shamsuddin, Finite normalizing extensions, J. Algebra 151 (1992), 498-499.

9. P. N. Stewart, Projective socles, Canad. Math. Bull. 32 (1989), 498-499.

10. P. N. Stewart and J. F. Watters, Properties of normalizing extensions and fixed rings, Comm. Algebra 12 (1984), 1067-1098.

11. Yufei Xiao, One sided SF-rings with certain chain conditions, Canad. Math. Bull. (to appear).

12. __ On rings whose flat cyclic modules are projective, preprint.

Department of Mathematics, University of Iowa, Iowa City, Iowa 52242

E-mail address: yxiao@oak.math.uiowa.edu 